\title{
Tree Traversal Synthesis Using Domain-Specific Symbolic Compilation
}

\author{
Yanju Chen \\ yanju@cs.ucsb.edu \\ University of California, Santa Barbara \\ USA \\ Yu Feng \\ yufeng@cs.ucsb.edu \\ University of California, Santa Barbara \\ USA
}

\author{
Junrui Liu \\ junrui@cs.ucsb.edu \\ University of California, Santa Barbara
}

USA

\author{
Rastislav Bodik \\ bodik@cs.washington.edu \\ University of Washington
}

USA

\begin{abstract}
Efficient computation on tree data structures is important in compilers, numeric computations, and web browser layout engines. Efficiency is achieved by statically scheduling the computation into a small number of tree traversals and by performing the traversals in parallel when possible. Manual design of such traversals leads to bugs, as observed in web browsers. Automatic schedulers avoid these bugs but they currently cannot explore a space of legal traversals, which prevents exploring the trade-offs between parallelism and minimizing the number of traversals.

We describe Hecate, a synthesizer of tree traversals that can produce both serial and parallel traversals. A key feature is that the synthesizer is extensible by the programmer who can define a template for new kinds of traversals. Hecate is constructed as a solver-aided domain-specific language, meaning that the synthesizer is generated automatically by translating the tree traversal DSL to an SMT solver that synthesizes the traversals. We improve on the general-purpose solver-aided architecture with a schedulingspecific symbolic evaluation that maintains the engineering advantages solver-aided design but generates efficient ILP encoding that is much more efficient to solve than SMT constraints.

On the set of Grafter problems, Hecate synthesizes traversals that trade off traversal fusion to exploit parallelism. Additionally, HecAte allows defining a tree data structure with an arbitrary number of children. Together, parallelism and data structure improvements accelerate the computation $2 \times$ on a tree rendering problem. Finally, HecATE's domain-specific symbolic compilation accelerates synthesis $3 \times$ compared to the general-purpose compilation to an SMT solver; when scheduling a CSS engine traversal, this ILP-based synthesis executes orders of magnitude faster.
\end{abstract}

\section{CCS CONCEPTS}

- Software and its engineering $\rightarrow$ Automatic programming.

\section{(c) (i)}

This work is licensed under a Creative Commons Attribution 4.0 International License.

ASPLOS '22, February 28 - March 4, 2022, Lausanne, Switzerland

(C) 2022 Copyright held by the owner/author(s)

ACM ISBN 978-1-4503-9205-1/22/02.

https://doi.org/10.1145/3503222.3507751

\section{KEYWORDS}

symbolic compilation, program synthesis, tree traversal

\section{ACM Reference Format:}

Yanju Chen, Junrui Liu, Yu Feng, and Rastislav Bodik. 2022. Tree Traversal Synthesis Using Domain-Specific Symbolic Compilation. In Proceedings of the 27th ACM International Conference on Architectural Support for Programming Languages and Operating Systems (ASPLOS '22), February 28 March 4, 2022, Lausanne, Switzerland. ACM, New York, NY, USA, 13 pages. https://doi.org/10.1145/3503222.3507751

\section{INTRODUCTION}

Traversal of tree structures is the foundation behind many applications: compilers leverage traversals of abstract syntax trees (ASTs) to analyze and optimize source codes. Layout engines in web browsers rely on traversals of render trees to determine the locations and appearances of HTML elements on web pages. Implementing tree traversals is a daunting task as it needs to strike a good balance between modularity and performance. On one hand, due to the complexity of modern layout engines, browser developers have to manually design scheduling strategies for rendering tree traversals in exchange for better performance. On the other hand, tree traversals in compilers are designed in a modular way, where mutually dependent traversals read and update attributes of ASTs [41]. This provides a great opportunity for automated scheduling of tree traversals. In particular, traversals that operate on the same node can be merged to reduce the overhead of node visiting and improve locality.

Even though manual scheduling of tree traversals offers finegrained control for maximizing performance of a layout engine, the complexity of layout semantics (e.g., from W3C CSS standards) make it difficult to maintain the infrastructure and fix the notorious bugs. For instance, the Servo layout engine contains several bugs that have been open for over five years $[14,25]$ due to a mismatch between the intended semantics and the architecture chosen for its implementation ${ }^{1}$.

Automated scheduling of tree traversals aims to merge modular passes (or visitors) that operate on the same node of a tree. However, existing approaches are far from satisfactory. For instance, there are approaches that are specialized to certain types of tree traversals,

\footnotetext{
${ }^{1}$ A Servo developer remarked that "it took three weeks before I realize[d] the actual complexity of the problem", which refers to the bug that is still open by the time of this submission; the Servo developers have resolved to delay fixing it until a complete rewrite of the layout engine is done [38].
} 
such as TreeFuser [40] and Grafter [41]. But they leverage deterministic rewrite rules as well as automata-based representations that are complex to maintain. Synthesis-based tools like FTL [32] express tree computations using attribute grammars and leverage constraint solvers (i.e., Prolog) to find candidate solutions that satisfy the dependencies among tree computations. However, FTL requires domain experts for translating the layout semantics into constraints in Prolog, which is error-prone.

Motivated by these observations, we introduce HecATE, a synthesizer of tree traversals that can produce both serial and parallel traversals. In particular, HECATE provides a high-level tree language for defining templates for new kinds of traversals. Furthermore, the core synthesis engine of HEcATE is built on top of a solver-aided framework [45], which lifts the execution of an interpreter for tree language programs into constraints that can be solved by off-theshelf solvers. As a result, HecATE eliminates the enormous engineering efforts in FTL while preserving the efficiency and flexibility of exploring different design choices. To use Hecate to synthesize a concrete traversal, the developer only needs to specify a simple traversal template with holes yet to be filled with computation rules defined by the tree language. After that, Hecate completes the traversals using a counterexample-guided inductive synthesis (CEGIS) loop [43]: the synthesizer searches for a candidate traversal that works for the initial examples. The verifier then looks for a counterexample that fails for the traversal and invokes the synthesizer again to find a new candidate that is consistent with the counterexample. The process continues until the verifier cannot find additional counterexamples.

As we show later in the evaluation, direct interpretation of full semantics of a tree traversal will lead to difficult-to-solve constraints due to path explosions. To address this, Hecate employs a domainspecific symbolic compilation strategy, which maintains the usability of symbolic compilation, yet scales to problems orders of magnitude larger. The key insight is a semantic projection layer between the interpreter and the symbolic compilation engine that tailors the constraint generation procedure. Specifically, we introduce a trace language that disentangles complex dependencies from time domain to relational domain, where constraints can be equivalently expressed independent of time, thus clearing out path explosions while still ensuring the correctness of constraints. Under domainspecific symbolic compilation, the trace language generates integer linear programming (ILP) constraints that can be solved efficiently.

We implement HecATE and compare it against GrafTER and FTL, showing that our tool is expressive, efficient, and flexible. On the set of Grafter problems, HecATE synthesizes traversals that trade off traversal fusion to exploit parallelism. Additionally, Hecate allows defining a tree data structure with an arbitrary number of children. Together, parallelism and data structure improvements accelerate the computation $2 \times$ on a tree rendering problem. Finally, HeCATE's domain-specific symbolic compilation accelerates synthesis $3 \times$ compared to the general-purpose compilation to an SMT solver; when scheduling a CSS engine traversal, this ILP-based synthesis executes orders of magnitude faster.

To summarize, we make the following contributions:

- We propose a CEGIS framework for tree traversals.

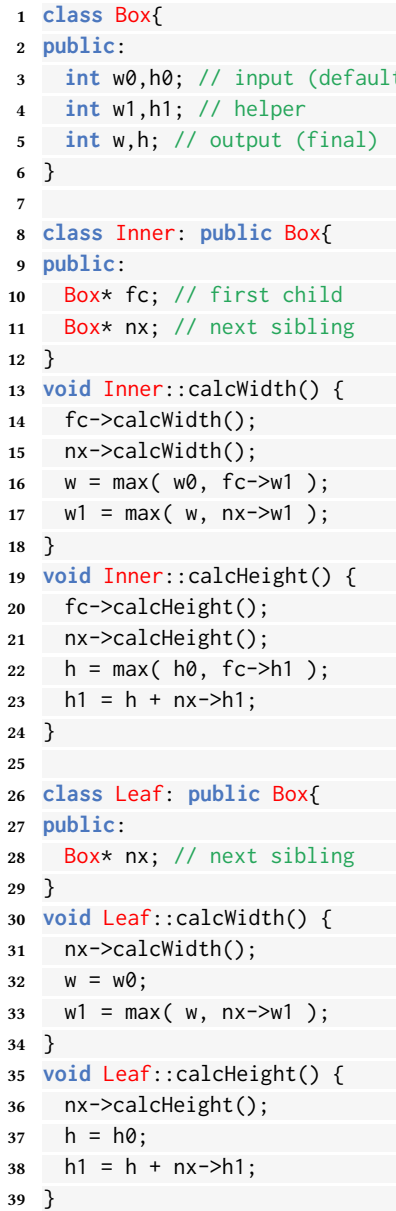

(a) unfused version

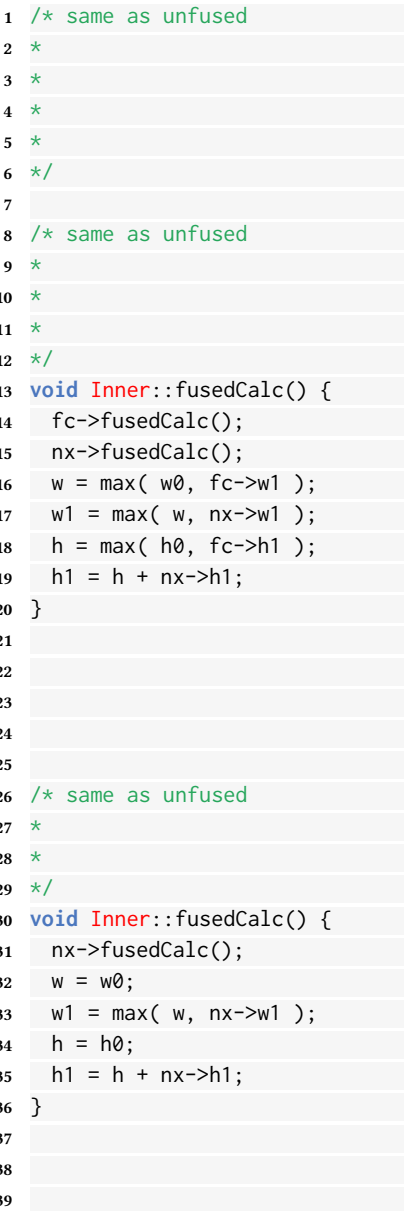

(b) fused version
Figure 1: Pseudo-code class definitions (unfused and fused versions) for rendering tree example.

- We propose a domain-specific trace language that disentangles complex dependencies from time domain to relational domain, which results in easy-to-solve constraints.

- We implement the proposed ideas in a tool called HecAte and demonstrate that it achieves $3 \times$ speed-up on GrAFTER benchmarks compared to general-purpose symbolic compilation.

\section{OVERVIEW}

In this section, we illustrate how HECATE works using a running example.

A Rendering Tree Example. Layout engines in modern web browsers utilize the box model in rendering procedures. This example demonstrates simplified behaviors of two types of boxes: Inner boxes and Leaf boxes where the former can hold child boxes and the latter can't. Figure 1(a) shows the realization of the boxes. In the example: 

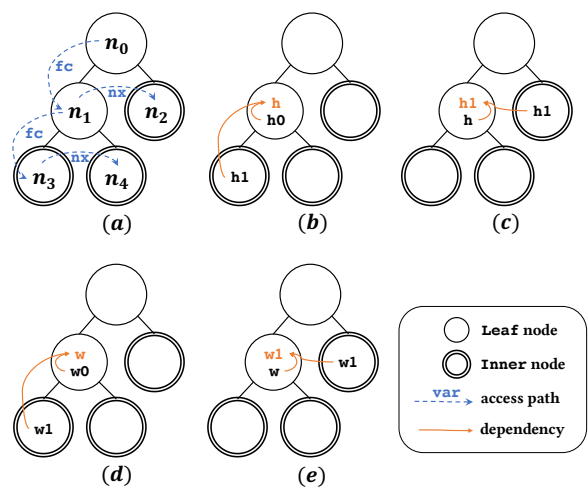

Figure 2: A motivating example tree with different properties shown (a): access paths; (b)-(e): dependencies between attributes of nodes.

- Line 16: The final width of an Inner box (denoted by w) is decided by the larger one between its default width (denote by wo) and the maximum width of its children (denoted by fc->w1).

- Line 22: The final height of an Inner box (denoted by h) is decided by the larger one between its default height (denoted by $\mathrm{h} 0$ ) and the summed height of its children (denoted by fc->h1).

- Line 33, 38: For a Leaf node, its final width and height are decided by its provided default values (denoted by wø and hø, respectively).

- Specifically, the helper variable w1 records the maximum final width among a node and all its siblings accessible by $\mathrm{nx}$, and the helper variable $h 1$ records the summed final height of a node and all its siblings accessible by nx. In other words, a node can refer to w1 of its first child as the maximum final width among all its children, and refer to h1 of its first child as the summed final height of all its children.

The two methods calcwidth and calcHeight demonstrate the computations for final width and height, respectively.

Typically, rendering a box requires a proper tree traversal to compute all the attribute values. Besides, in real-world use case scenarios, a finer-grained scheduling of computations is usually required as an optimization. As shown in Figure 1(b), a more efficient method fusedCalc is synthesized to perform width and height computations at the same time. Because attribute values may depend upon one another, solving for a correct order of attribute evaluations becomes challenging.

How Hecate Works. Now with Hecate, the user starts by providing: 1) a symbolic traversal as shown in Figure 4(a) with slots $t_{i}$ in which we can schedule at most one computation rule from the grammar, 2) an example tree shown in Figure 2(a), and 3) the corresponding semantics written in HECATE's visitor language (shown in Figure 3). HecAte then automatically synthesizes a concrete traversal by filling the slots with computation rules while respecting all the read-write dependencies, as shown in Figure 4(b).

More specifically, executing the traversal over the example tree on an Inner node (e.g., $n_{1}$ ) first recursively computes the attributes

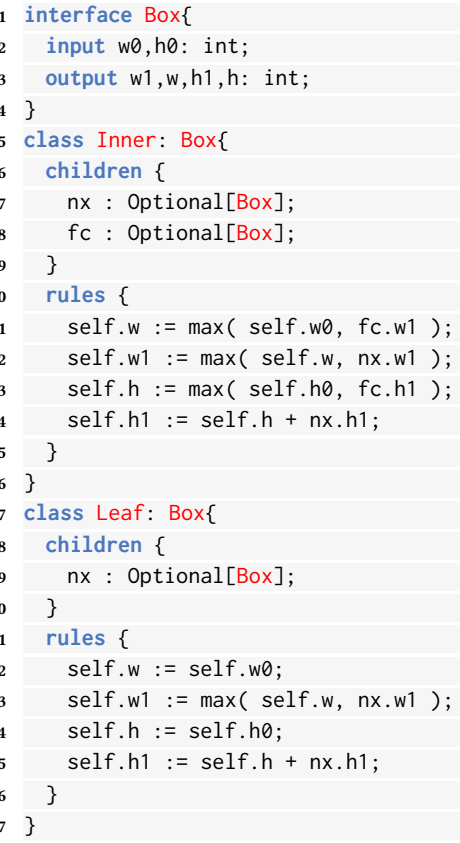

Figure 3: Class definitions in HecATE for rendering tree example.

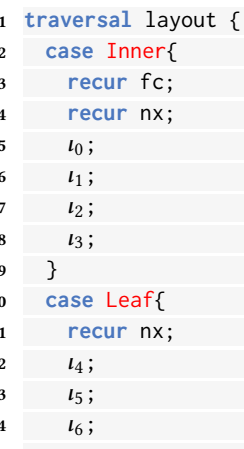

(a) symbolic

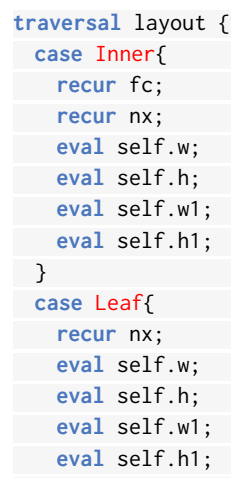

\section{(b) concrete}

Figure 4: Symbolic and concrete tree traversals for rendering tree example.

of its child nodes (line 3 in Figure 4(b), i.e., $n_{3}$ and $n_{4}$ in Figure 2(a)) and its next sibling (line 4, i.e., $n_{2}$ ), and then attributes of itself (line 5-8). For a Leaf node, the computation is similar but without the recursion on the children.

Figure 5 illustrates the overview of HecATE, which instantiates a CEGIS loop with two phases: 1) Given the specification that contains class definitions as attribute grammar, initial trees, and a symbolic traversal, the synthesizer searches for a concrete traversal, and sends it to the verifier; 2) The verifier checks the correctness of the concrete traversal over all possible example trees (up to depth $k$ ) 


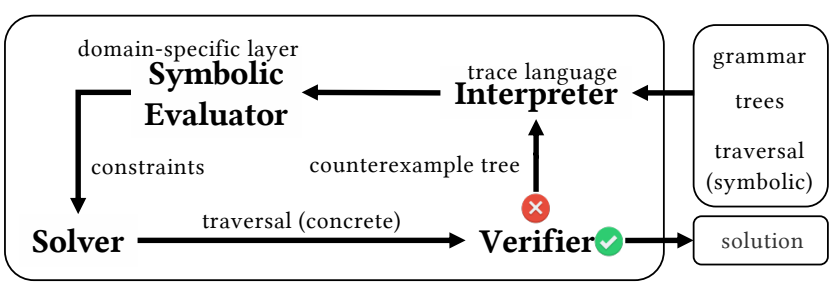

Figure 5: Overview of Hecate.

and returns a counterexample tree that fails the current traversal. Then the synthesizer finds a new candidate that is consistent with the newly added counterexample tree. This process continues until the verifier can not find additional counterexamples to refute the current traversal, which will be returned as the correct solution.

To synthesize the desired traversal, Hecate leverages Rosette [45] to lift an interpreter for tree traversal into a synthesizer through symbolic compilation. In particular, given a symbolic traversal with slots, symbolic compilation expands each slot into all possible choices of computation rules, and executes the traversal to generate dependency constraints under different choices. A concrete traversal is then obtained by solving the constraints using off-the-shelf SMT solvers [18, 24].

While a faithful interpretation of the semantics of the traversal usually causes path explosions, as choices of rules depend on choices made on preceding execution steps, it generates complex SMT constraints that are hard to solve, thus damaging the performance. Our solution is to insert a domain-specific layer below the interpreter and above the symbolic engine (i.e., RosETTE), which exposes a domain-specific trace language that still allows us to write the interpreter conveniently, while avoiding explicit path conditions and generating compact ILP constraints that are easier to solve by off-the-shelf ILP solvers [24].

\section{PROBLEM FORMULATION}

In order to precisely describe our synthesis problem, we first present some definitions that we use throughout the paper.

\subsection{Attribute Grammar for Tree Visitors}

To represent tree structures and their visitors that contain a set of computation rules over attributes, we introduce a tree visitor language $\mathcal{L}_{a}$ based on attribute grammar [26]. Figure 6 defines the syntax of $\mathcal{L}_{a}$ in an object-oriented style:

- A class (i.e., $\langle$ class $\rangle$ ) represents the type(s) of a tree node.

- A node can refer to its child nodes via 〈children $\rangle$ block, and each node also stores values of primitive fields - we call these fields attributes.

- A $\langle$ rules $\rangle$ block contains computation rules and each rule is a $\langle c s t m t\rangle$ statement.

- The left-hand-side (LHS) of a statement specifies an access path $\langle$ sel $\rangle$ to an attribute that is computed by the expression in the right-hand-side (RHS).

- An expression on the RHS can be: constants, binary expressions, branches, aggregations, and function calls, etc.

- Each attribute can be assigned exactly once.

$$
\begin{array}{ll}
\langle\text { interface }\rangle & ::=\text { interface }\langle\text { id }\rangle\left\{(\langle\text { tup }\rangle ;)^{*}\right\} \\
\langle\text { class }\rangle & ::=\text { class }\langle\text { tup }\rangle\{\langle\text { children }\rangle\langle\text { rules }\rangle\} \\
\langle\text { children }\rangle & ::=\text { children }\left\{(\langle\text { tup }\rangle ;)^{*}\right\} \\
\langle\text { rules }\rangle & ::=\text { rules }\left\{(\langle\text { cstmt }\rangle ;)^{*}\right\} \\
\langle\text { tup }\rangle & ::=\langle\text { id }\rangle:\langle\text { id }\rangle(,\langle\text { id }\rangle)^{*} \\
\langle\text { sel }\rangle & ::=\langle\text { id }\rangle(.\langle\text { id }\rangle) ? .\langle\text { id }\rangle \\
\langle\text { expr }\rangle & ::=\langle\text { const }\rangle \mid\langle\text { sel }\rangle \mid f\left(\langle\text { expr }\rangle^{*}\right) \\
& \mid \text { expr }\rangle\langle\text { op }\rangle\langle\operatorname{expr}\rangle \mid \text { fold }(\langle\text { expr }\rangle+) \\
& \mid \text { if }\langle\text { expr }\rangle \text { then }\langle\text { expr }\rangle \text { else }\langle\text { expr }\rangle \\
\langle\text { cstmt }\rangle & ::=\langle\text { sel }\rangle:=\langle\text { expr }\rangle \\
\langle\text { op }\rangle & ::=+|-| \times|\div| \ldots \\
\quad f \in \text { functions } & \langle\text { const }\rangle \in \text { constants }\langle\text { id }\rangle \in \text { identifiers }
\end{array}
$$

Figure 6: Syntax for attribute grammar $\mathcal{L}_{a}$.

$$
\begin{array}{ll}
\langle\text { traversal }\rangle & ::=\operatorname{traversal}\langle\text { id }\rangle\left\{\langle\text { case }\rangle^{*}\right\} \\
\langle\text { case }\rangle & ::=\text { case }\langle\text { id }\rangle\left\{(\langle\text { tstmt }\rangle ;)^{*}\right\} \\
\langle\text { recur }\rangle & ::=\operatorname{recur}\langle\text { node }\rangle \\
\langle\text { iterate }\rangle & ::=\text { iterate }\left\{(\langle\text { tstmt }\rangle ;)^{*}\right\} \\
\langle\text { parallel }\rangle & ::=\operatorname{parallel}\left\{(\langle\text { tstmt }\rangle ;)^{*}\right\} \\
\langle\text { eval }\rangle & ::=\operatorname{eval}\langle\text { cstmt }\rangle \\
\langle\text { tstmt }\rangle & ::=\iota \mid\langle\text { recur }\rangle \mid\langle\text { iterate }\rangle \mid\langle\text { eval }\rangle
\end{array}
$$$$
\langle i d\rangle \in \text { identifiers }\langle\text { node }\rangle \in \text { nodes }
$$

Figure 7: Syntax for tree traversal language $\mathcal{L}_{t}$.

As a result, $\mathcal{L}_{a}$ is specialized for modeling the behaviors of reading and writing of attributes of the current node and its children, which essentially describes the dependency relations between attributes of nodes.

Example 3.1. The code snippet from Figure 3 declares the attribute grammar for nodes of types Inner and Leaf, which share the same set of attributes declared by the interface Box. Specifically:

- each Inner node has two children $n x$ and fc that point to its next sibling and first child respectively;

- a Leaf node does not have children.

Rules for computing the attributes vary across different classes. e.g.,

- attributes $w$ and $h$ of a Leaf node only depend on attributes from itself

- attribute $w$ from an Inner node depends on the default width of itself (i.e., self .w $\bullet$ ) and the maximum width of its children (i.e., fc.w1),

- attribute $\mathrm{h}$ from an Inner node depends on the default height of itself (i.e., self.h $\theta$ ) and the summed height of its children (i.e., fc.h1).

\subsection{Language for Tree Traversals}

To formally define a tree traversal, we first introduce domains for different notations.

Syntax. Figure 7 summarizes the language $\mathcal{L}_{t}$ for expressing tree traversals. In particular, A traversal $\langle$ traversal $\rangle$ is declared with a list of $\langle$ case $\rangle$ blocks. Each $\langle$ case $\rangle$ block matches a node type and contains statements of the following forms: 
- $\langle$ recur $\rangle$ recursively visits a child of the current node,

- 〈iterate〉 iteratively visits every child of the current node,

- $\langle$ parallel $\rangle$ in parallel visits every child of the current node ${ }^{2}$,

- $\langle$ eval $\rangle$ evaluates an attribute computation rule, and

- a slot $\iota$ represents zero or one computation rule yet to be determined ${ }^{3}$.

Domains. Our system contains the following domains:

- Each traversal operates over a set of trees denoted by $\mathbb{E}$.

- Each tree contains a set of nodes (i.e., $\mathbb{N}$ ).

- A time domain $t \in \mathbb{T}$ enforces the order of different rules.

- An attribute grammar provides a list of attributes $\mathbb{A}$ that uniquely determine their corresponding computation rules.

- Each traversal provides a list of slots (i.e., I) for holding computation rules from the attribute grammar.

- Each node $n$ has a set of locations (i.e., $\mathbb{L}$ ) that refer to its corresponding attributes in runtime.

Definition 3.2. Traversal. Given a tree, a traversal defines a total order relation $\prec$ over the set of all locations of the tree.

Example 3.3. A concrete post-order traversal (i.e., Figure 4(b)) on the tree in Figure 2 yields the following total order of locations:

$$
\begin{aligned}
n_{4} \cdot \mathrm{w} & <n_{4} \cdot \mathrm{h}<n_{4} \cdot \mathrm{w} 1<n_{4} \cdot \mathrm{h} 1<n_{3} \cdot \mathrm{w}<n_{3} \cdot \mathrm{h}<n_{3} \cdot \mathrm{w} 1<n_{3} \cdot \mathrm{h} 1 \\
<n_{1} \cdot \mathrm{w} & <n_{1} \cdot \mathrm{h}<n_{1} \cdot \mathrm{w} 1<n_{1} \cdot \mathrm{h} 1<n_{2} \cdot \mathrm{w}<n_{2} \cdot \mathrm{h}<n_{2} \cdot \mathrm{w} 1<n_{2} \cdot \mathrm{h} 1 \\
<n_{0} \cdot \mathrm{w} & <n_{0} \cdot \mathrm{h}<n_{0} \cdot \mathrm{w} 1<n_{0} \cdot \mathrm{h} 1
\end{aligned}
$$

where in every time step $t \in \mathbb{T}$ one location is visited. Note that different traversals may induce different orders.

A traversal is symbolic if it contains at least one slot $\iota$ and is concrete otherwise.

Example 3.4. Figure 4(a) declares a symbolic and post-order traversal over nodes of types Inner and Leaf. Figure 4(b) is an instantiation of the previous symbolic traversal. In particular, the traversal first computes the attributes for the leaves of type Leaf, and then the attributes of the nodes of type Inner.

Note that the concrete post-order traversal preserves the readwrite dependencies induced by the attribute grammar in Figure 3. On the contrary, a pre-order traversal would not be valid since it violates the read-write dependencies imposed by attributes including $\mathrm{w}$ and $\mathrm{h}$ of a Inner node. We then define the tree traversal synthesis problem as follows:

Definition 3.5. Tree Traversal Synthesis. Given an attribute grammar $\mathcal{L}_{a}$ and a symbolic traversal $\hat{P}_{t}$ with holes, a tree traversal synthesis problem is to induce a concrete traversal $P_{t}$ by completing the holes $\iota$ in $\hat{P}_{t}$ with computation rules in $\mathcal{L}_{a}$, such that for arbitrary instantiated trees from $\mathcal{L}_{a}: 1$ ) all attributes are computed, and 2) all read-write dependencies are preserved.

Example 3.6. Given the attribute grammar $\mathcal{L}_{a}$ in Figure 3 and the symbolic traversal in Figure 4(a), Hecate synthesizes the concrete traversal in Figure 4(b). Given arbitrary tree derived from Figure 2, the synthesized traversal computes all attributes of the tree exactly once and respects all read-write dependencies.

\footnotetext{
${ }^{2}$ If parallelism is impossible, HecAte falls back to $\langle$ iterate $\rangle$.

${ }^{3}$ One can extends the semantics of $\iota$ to represent one or more rules.
}

As we show later, to bypass the challenge of complex SMT constraints that are generated by a faithful interpretation of a traversal's semantics, a scalable approach to solve the placement and resource allocation problems is to use ILP to map the computation rules to the available slots in the traversal $[15,36]$.

Definition 3.7. 0-1 Integer Linear Programming. Given coefficients $a, b$ and $c$, the 0-1 ILP problem is to solve for $x$ as follows:

$$
\min \sum_{i} c_{i} x_{i} \quad \text { s.t. } \forall a_{i, j} \cdot \sum_{j} a_{i, j} x_{j} \leq b_{i},
$$

where all entries are integers and in particular $x_{j} \in\{0,1\}$.

We obtain a set of ILP constraints that is easy to solve by ILP solvers from domain-specific symbolic compilation via program written in trace language, which is deferred to Section 5 for a detailed discussion.

\section{TREE TRAVERSAL SYNTHESIS}

In this section, we formally introduce our synthesis framework for tree traversals that is based on counterexample-guided inductive synthesis (CEGIS). In what follows, we first give a high-level overview of the synthesis framework, then we show how to reduce the synthesis problem to a general-purpose symbolic compilation problem based on Rosette. Finally, we briefly discuss its limitation.

\subsection{System Overview}

As shown in Figure 5, HecAte takes as inputs an attribute grammar $\mathcal{L}_{a}$, a symbolic traversal with unknown slots in $\mathcal{L}_{t}$, and an initial tree for validating the correctness of the traversal. The output of HECATE is a concrete traversal that respects all the read-write dependencies imposed by the attribute grammar.

Synthesis. Figure 8(a) sketches the core synthesis engine that is built on top of RosetTe [45], a hybrid symbolic compiler that combines symbolic execution and bounded model checking to compute compact constraints. In particular, the general-purpose interpreter interpret for tree traversals takes as inputs a grammar grammar, a traversal traversal, and a concrete tree tree. Following the total order induced by traversal, the outermost loop of the interpreter recursively visits each node in tree and its corresponding locations loc (line 2). When evaluating a symbolic choice for a slot, symbolic evaluation considers each alternative concrete rule (line 3-4), generates the constraints stating that the dependencies are ready and the target has not been computed (line 6-7), sets the target attribute as ready, and updates the program state (line 8 ). The interpreter behaves as a regular emulator when it runs with concrete traversals and trees. For instance, running it with the post-order traversal in Figure 4(b) and the example tree in Figure 2 will pass all assertions and terminate normally. What is more interesting is that given a symbolic traversal traversal with slots yet to be filled, RoseTTE runs the interpreter with traversal and a concrete tree tree under symbolic evaluation; this encodes all possible concrete traversals that preserve the dependencies in tree, effectively lifting the interpreter to be a synthesizer. 


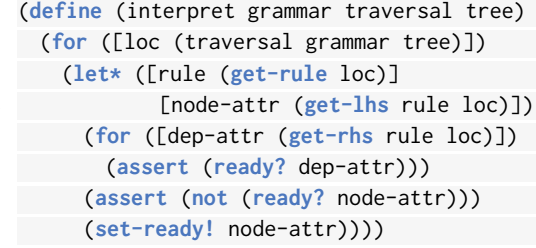

\section{(b) domain-specific interpreter}

Figure 8: Code snippets of general-purpose interpreter and domain-specific interpreter.

Verification. To ensure that the synthesized traversal traversal is not only correct on the initial example tree but also on all possible trees ${ }^{4}$, we again leverage RosetTe to build our verifier. In particular, the core of the verifier is another interpreter that is almost identical to the one in Figure 8(a). Now the inputs of the interpreter include a concrete traversal traversal that needs to be verified, as well as a symbolic tree $\hat{r} r$ that encodes the space of all possible concrete examples up to depth $k$. In that case, symbolically evaluating $\hat{t r}$ on traversal yields a formula stating that tree is correct on all instantiations of $\hat{t r}$. If the formula is satisfiable, the verifier then returns a counterexample to the synthesizer that will look for another candidate. Similar to prior work in Neo [20] and BonsAI [15], our symbolic tree $\hat{t r}$ is encoded as a bounded $m$-ary tree derived from the attribute grammar. We omit the details since it is not the main contribution.

We call the interpreter in Figure 8(a) general-purpose symbolic compilation. By nature, its encoding (general-purpose encoding) establishes read-write dependencies across different execution time steps. While general-purpose encoding is fairly intuitive and straightforward to implement, it may lead to complex constraints that are difficult to solve.

\subsection{General-Purpose Symbolic Compilation}

In this section, we elaborate on the details behind general-purpose symbolic compilation. Using the domains introduced in Section 3.2, we first define a set of relational operators to formalize the generalpurpose symbolic compilation:

- Assignment. The assignment operator maps an attribute $a \in$ $\mathbb{A}$ and a slot $\iota \in \mathbb{I}$ to a boolean variable in $\mathbb{B}$, i.e., $\sigma: \mathbb{A} \times \mathbb{I} \rightarrow \mathbb{B}$. Since each attribute $a$ is uniquely computed by a computation rule from the attribute grammar, predicate $\sigma(a, l)$ evaluates to true iff the rule for computing attribute $a$ is scheduled at slot $\iota$.

\footnotetext{
${ }^{4}$ Similar to prior work [15], we verify trees up to depth $k$.
}

- Dereference. Recall that in Figure 6, each computation rule for an attribute is composed of multiple access paths sel appearing in a statement. During tree traversal, the dereference operator $\zeta(n, \mathrm{sel})$ returns the concrete location $l$ (i.e., $\mathbb{N} \times \mathbb{A}$ ) that access path sel of node $n$ points to.

- Ready Bit. The ready bit operator maps a node $n \in \mathbb{N}$, an attribute $a \in \mathbb{A}$, and a time step $t \in \mathbb{T}$ to a boolean variable in $\mathbb{B}$, i.e., $\delta: \mathbb{N} \times \mathbb{A} \times \mathbb{T} \rightarrow \mathbb{B}$. Here, predicate $\delta(n, a, t)$ returns true iff the attribute $a$ of node $n$ is already computed (i.e., ready for being read by other computation rules) before time step $t$.

- Symbolic Choice. Recall that a symbolic traversal $\hat{P}_{t}$ contains at least one slot $\iota$ that represents at most one attribute computation yet to be scheduled. To handle this case, we introduce a symbolic choice operator choose for non-deterministically choosing an attribute (to compute) from a list of available attributes $\mathbb{A}$. For instance, (choose [Inner.h, Inner.w, none]) returns one of the attributes from the list.

Now, during the general-purpose symbolic compilation in Figure $8(\mathrm{a})$, the interpreter executes statements in traversal trav and dynamically inserts assertions (line 6-7) to state the correctness of every single computation rule. In particular, the correctness enforces read-write dependency using the ready bit operator $\delta$. Therefore, for a statement with chosen attribute, e.g., eval self.h at slot $\iota_{2}$ of node $n_{1}$, RosetTe compiles it into the following constraints:

$$
\delta\left(\zeta\left(n_{1}, \text { self.h } \theta\right), t\right) \wedge \delta\left(\zeta\left(n_{1}, \text { fc.h1 }\right), t\right) \wedge \neg \delta\left(\zeta\left(n_{1}, \text { self.h }\right), t\right)
$$

where $t$ is the current time step and rule self.h $:=\max (\operatorname{self} . h 0$, fc.h1 ) (line 13 in Figure 3) is used to compute attribute self.h for node $n_{1}$ of type Inner. Here, the above constraints state two properties about the read-write dependencies: 1) attributes of nodes (i.e., self.h $\theta$ for $n_{1}$ and fc.h1 for $n_{3}$ ) should be ready before they are read, and 2) the attribute of a node (i.e., self.h for $n_{1}$ ) should not be ready until it is written.

The interpreter starts by executing a slot statement $\iota$ in the symbolic traversal. In that case, each $\iota$ is dynamically replaced by a statement that non-deterministically chooses an available attribute $a_{i}$ to schedule ${ }^{5}$ : eval (choose $\left[a_{1}, \ldots, a_{n}\right]$ ). After that, Rosetre symbolically evaluates the above statement and compiles it into a formula stating all possible cases where each case is guarded by the conjunction of assignment operators $\sigma$ that represent the cumulative choices so far.

Example 4.1. For instance, at time step $t$, when the interpreter executes slot $\iota_{2}$, i.e. line 7 in Figure 4(a), on Inner node $n_{1}$ in Figure 10(a), it can choose one of the five options from none, Inner.w1, Inner. w, Inner. $h 1$ and Inner. $h$ according to its attribute grammar in Figure 10(b):

$$
\text { eval (choose S) }
$$

where

$$
\text { S : [none, Inner.w1, Inner.w, Inner.h1, Inner.h] }
$$

\footnotetext{
${ }^{5}$ We omit the empty case for simplicity.
} 
which is further transformed into the following formula:

$$
\begin{aligned}
\left(\sigma\left(\text { none, } \iota_{2}\right)\right. & \Longrightarrow \text { true }) \\
\vee\left(\sigma\left(\text { Inner.w1, } \iota_{2}\right)\right. & \Longrightarrow \delta\left(\zeta\left(n_{1} \text {, self.w }\right), t\right) \wedge \delta\left(\zeta\left(n_{1}, \mathrm{nx} . \mathrm{w} 1\right), t\right) \\
& \left.\wedge \neg \delta\left(\zeta\left(n_{1} \text {, self.w1 }\right), t\right)\right) \\
\vee\left(\sigma\left(\text { Inner.w, } \iota_{2}\right)\right. & \Longrightarrow \delta\left(\zeta\left(n_{1} \text {, self.w }\right), t\right) \wedge \delta\left(\zeta\left(n_{1}, \mathrm{fc} . \mathrm{w} 1\right), t\right) \\
& \left.\wedge \neg \delta\left(\zeta\left(n_{1} \text {, self.w }\right), t\right)\right) \\
\vee\left(\sigma\left(\text { Inner.h1, } \iota_{2}\right)\right. & \Longrightarrow \delta\left(\zeta\left(n_{1} \text {, self.h }\right), t\right) \wedge \delta\left(\zeta\left(n_{1}, \mathrm{nx} . \mathrm{h} 1\right), t\right) \\
& \left.\wedge \neg \delta\left(\zeta\left(n_{1} \text {, self.h1 }\right), t\right)\right) \\
\vee\left(\sigma\left(\text { Inner.h, } \iota_{2}\right)\right. & \Longrightarrow \delta\left(\zeta\left(n_{1} \text {, self.h }\right), t\right) \wedge \delta\left(\zeta\left(n_{1}, \mathrm{fc} . \mathrm{h} 1\right), t\right) \\
& \left.\wedge \neg \delta\left(\zeta\left(n_{1}, \text { self.h }\right), t\right)\right)
\end{aligned}
$$

where $\sigma$ (Inner.h, $l_{2}$ ) evaluates to true iff we decide to compute attribute Inner. $\mathrm{h}$ at slot $\iota_{2}$ using its corresponding rule self. $\mathrm{h}:=$ $\max ($ self.h0, fc.h1) from class Inner. Here in particular, every assignment predicate $\sigma$ implies a conjunction of three ready bit predicates $\delta$ asserting corresponding properties of read-write dependencies. For example, the last clause

$$
\begin{aligned}
\sigma\left(\text { Inner.h, } \iota_{2}\right) & \Longrightarrow \delta\left(\zeta\left(n_{1}, \text { self.h } 0\right), t\right) \wedge \delta\left(\zeta\left(n_{1}, \text { fc.h1 }\right), t\right) \\
& \wedge \neg \delta\left(\zeta\left(n_{1}, \text { self.h }\right), t\right)
\end{aligned}
$$

indicates that in order to schedule rule Inner. $h$ at slot $\iota_{2}$,

- self.h 0 and fc.h1 should be ready before time step $t$, and

- self.h should not be scheduled before time step $t$.

In addition to correctness constraints, we also enforce auxiliary constraints to induce valid traversals. For instance, the following constraint requires every slot be filled with at most one rule:

$$
\forall \iota .\left(\bigvee_{a_{0}} \bigwedge_{a \neq a_{0}} \neg \sigma(a, \iota) \wedge \sigma\left(a_{0}, \iota\right)\right) \vee\left(\bigwedge_{a} \neg \sigma(a, \iota)\right) .
$$

And the following requires every rule be used by only one slot:

$$
\forall a . \bigvee_{\iota_{0}} \bigwedge_{\iota \neq \iota_{0}} \neg \sigma(a, \iota) \wedge \sigma\left(a, \iota_{0}\right)
$$

Performance Analysis. While it is intuitive and straightforward to build a tree traversal synthesizer using general-purpose encoding, it suffers from path explosion by faithfully following the execution of a traversal, even with the effective state-merging and pruning strategy from RosetTE. Figure 9 shows how the number of symbolic state grows as time goes by. Consider a tree of $n$ nodes with an average of $k$ slots per node, the general-purpose symbolic compilation will generate constraints based on a chain of length $n \cdot k$ with dependencies between choices made in a recursive way, i.e. nested choose operations. Assuming that every slot has a candidate set of $q$ rules to fill in on average, the total number of symbolic states after compilation can be up to $q^{n \cdot k}$. As shown in our evaluations, the general-purpose symbolic compilation creates constraints that take a long time to solve.

\section{DOMAIN-SPECIFIC SYMBOLIC COMPILATION}

As discussed in Section 4, a general-purpose symbolic compilation faithfully follows the execution of a traversal across different execution time steps, which leads to constraints that are hard to solve. To mitigate this problem, we propose a domain-specific trace language, which projects the complex dependencies from time domain to relational domain and yields easy-to-solve constraints. In what follows, we first introduce the trace language $\mathcal{L}_{r}$, and then

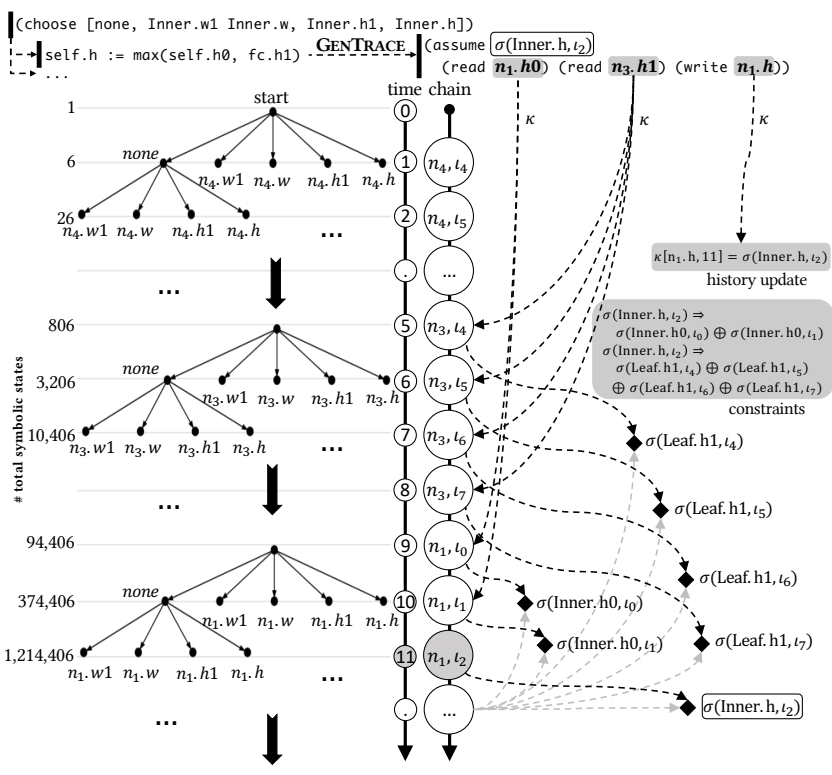

Figure 9: General-purpose (left) v.s. domain-specific (right) symbolic compilations.

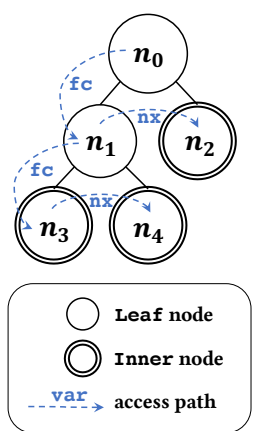

(a) example tree

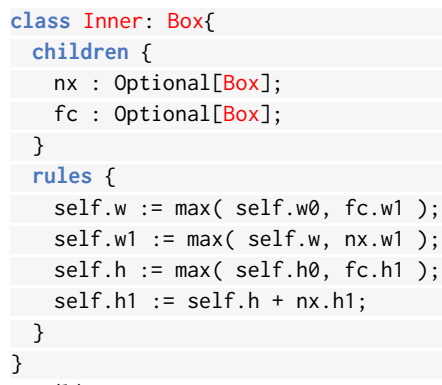

(b) visitor program snippet
Figure 10: The motivating example tree as in Section 2 and its corresponding visitor program snippet.

show how to obtain ILP constraints via domain-specific symbolic compilation.

\subsection{A Trace Language for Tree Traversals}

As shown in Figure 8(b), thanks to RosetTe, the skeleton of domain-specific interpreter for synthesizing tree traversals can be obtained with a minor modification over the general-purpose version: upon executing a statement in traversal traversal, instead of directly adding its corresponding assertions, we first translate the statement into another program in trace language $\mathcal{L}_{r}$, and then leverage RosetTe to lift the execution of the new trace program to constraints that can be modeled as an integer linear programming (ILP) problem. 
Table 1: Operations of the symbolic trace language $\mathcal{L}_{r}$.

\begin{tabular}{|l|l|}
\hline Operation & Description \\
\hline \hline (choose $\left.\left[a_{1}, \ldots a_{n}\right]\right)$ & choose one from the attributes \\
\hline (alloc) & returns a fresh concrete location \\
\hline$($ read $n . a)$ & logs a read from $n . a$ \\
\hline (write $n . a)$ & logs a write to n.a \\
\hline
\end{tabular}

The syntax and semantics of $\mathcal{L}_{r}$ are summarized in Table 1. Intuitively, $\mathcal{L}_{r}$ understands dependency relations carried through attributes on nodes with fully abstract contents. In particular:

- (read n.a) logs the read action of attribute $a$ on node. $n$;

- (write n.a) logs the write action of attribute $a$ on node $n$.

- (choose $\left[a_{1}, \ldots, a_{n}\right]$ ) non-deterministically selects an attribute $a_{i}$ to compute.

For the sake of simplicity, we use the built-in assume function in RosETTE to explicitly enumerate each option of symbolic choices under different assumptions.

During the execution of the domain-specific interpreter (Figure 8(b)) at line 6, HecATE invokes a syntax-directed transpilation procedure to generate the corresponding trace program, which captures the dependency relations to ensure the correctness of tree traversals, and provides succinct statements that eventually lead to efficient constraints (Section 5.2).

Example 5.1. Following Example 4.1, suppose we are in the symbolic traversal (i.e., Figure 4(a)) at slot $l_{3}$ and the current node is $n_{0}$ in Figure 10(a). And the synthesizer decides to select a rule self.h $:=\max ($ self.h0, fc.h1) from the visitor program in Figure 10(b) to compute the Inner. $h$ attribute in slot $\iota_{3}$, then a syntax-directed transpilation procedure is invoked to generate the following trace program:

(assume $\sigma\left(\right.$ Inner.h, $\left.\iota_{3}\right) \quad\left(\right.$ read $\left.n_{0} \cdot \mathrm{h} 0\right)\left(\right.$ read $\left.n_{1} \cdot \mathrm{h} 1\right) \quad\left(\right.$ write $\left.\left.n_{0} \cdot \mathrm{h}\right)\right)$.

Semantically the above trace program states that in order to compute Inner.h at slot $\iota_{3}$, two attributes (i.e., $n_{0} . \mathrm{h} 0$ and $n_{1}$.h1) should first be read and another attribute (i.e., $\left.n_{0} . h\right)$ should then be written. The trace program records read-write dependencies in a more compact way without introducing time steps.

\subsection{Symbolic Compilation of Trace Program}

Even if we obtain a trace program $P_{r}$ using the procedure discussed in Section 5.1, the trace program itself does not mitigate the path explosion problem because similar to the general-purpose encoding, the symbolic choice statements in the trace program still encode path conditions at each time step. To address this challenge, we discuss how our domain-specific compilation further projects the executions of the trace program into compact constraints that can be solved by efficient ILP solvers [24].

Dependency Constraints. We first introduce a dependency operator $\kappa$ that takes as inputs a location $l \in \mathbb{N} \times \mathbb{A}$, a time step $t \in \mathbb{T}$, and returns a boolean variable $\sigma$ that specifies all possible slots in which the attribute $a$ of location $l$ was computed. In other words, $\kappa$ encodes the dependency between locations (i.e., read) and their corresponding attributes (i.e., write).
We then illustrate the relationship between the trace program and the dependency operator $\kappa$. In particular, for a write instruction (write n.a) at time step $t$ guarded by $\sigma(a, l)$, the dependency operator $\kappa$ gets updated by:

$$
\kappa(n . a, t) \leftarrow \sigma(a, \iota),
$$

where attribute $a$ is written at time step $t$ if $\sigma(a, l)$ evaluates to 1 (i.e., true).

For a read instruction (read n.a) at time step $t$ guarded by $\sigma(a, l)$, if $\sigma(a, \iota)$ evaluates to true, then it implies that attribute $a$ must be written somewhere before time step $t$. Formally speaking, we have constraint:

$$
\sigma(a, \iota) \Longrightarrow\left(\exists t_{0} \cdot\left(t_{0}<t\right) \wedge \kappa\left(n . a, t_{0}\right)\right),
$$

which can be easily translated into its equivalent ILP constraint ${ }^{6}$ :

$$
\sigma(a, \iota) \leq \sum_{t_{0}<t} \kappa\left[n \cdot a, t_{0}\right], \quad \quad \text { (read constraint) }
$$

Example 5.2. Following Example 4.1 but in domain-specific symbolic compilation, as shown in Figure 9, suppose we want to schedule self.h $:=\max$ ( self.h0, fc.h1 ) at slot $\iota_{2}$ of node $n_{1}$, which corresponds to the following trace program:

(assume $\sigma\left(\right.$ Inner.h, $\left.\iota_{2}\right) \quad\left(\right.$ read $\left.n_{1} . h 0\right)\left(\right.$ read $\left.n_{3} . h 1\right)$ (write $\left.\left.n_{1} . \mathrm{h}\right)\right)$.

The domain-specific encoding compiles the above trace program into the following ILP constraints:

$$
\begin{aligned}
\sigma\left(\text { Inner.h, } \iota_{2}\right) & \leq \sum_{t_{0}<t} \kappa\left[n_{1} \cdot \mathrm{h} \emptyset, t_{0}\right] \\
& =\sigma\left(\text { Inner.h } \theta, \iota_{0}\right)+\sigma\left(\text { Inner.h } \theta, \iota_{1}\right), \quad\left(\text { read for } n_{1} \cdot \mathrm{h} \theta\right) \\
\sigma\left(\text { Inner.h, } \iota_{2}\right) & \leq \sum_{t_{0}<t} \kappa\left[n_{3} . \mathrm{h} 1, t_{0}\right] \\
& =\sigma\left(\text { Leaf.h1, } \iota_{4}\right)+\sigma\left(\text { Leaf.h1, } \iota_{5}\right) \\
& +\sigma\left(\text { Leaf.h1, } \iota_{6}\right)+\sigma\left(\text { Leaf.h1 }, \iota_{7}\right), \quad\left(\text { read for } n_{3} . \mathrm{h} 1\right)
\end{aligned}
$$

where $t$ corresponds to the time step when visiting $\iota_{2}$ of node $n_{1}$. According to Definition 3.2, since a traversal defines a total order relation over all locations of a tree, we can map the location that is currently being evaluated to a certain time step $t$, and generate constraints that require all the dependencies of this location are ready before time step $t$. This is done by $\kappa$ in the example. Then, we again utilize the mapping to cancel the time step variables in the constraints by mapping them back to potential locations, thus resulting in a more compact constraint system.

Validity Constraints. Similar to the general-purpose encoding in Section 4.2 , we also impose extra constraints to ensure the validity of the traversals.

- For every slot $\iota$, at most one rule can be filled in:

$$
\forall \iota . \sum_{a} \sigma(a, \iota) \leq 1, \quad \text { (slot constraint) }
$$

- Every rule $a$ is used by exact one slot $l$ :

$$
\forall a . \sum_{\iota} \sigma(a, \iota)=1
$$

(rule constraint)

\footnotetext{
${ }^{6}$ Interchangeably, we use the same domain notation B to denote the boolean domain and 0-1 integer domain for general-purpose and domain-specific symbolic encodings, respectively.
} 
Performance Analysis. To understand why the domain-specific compilation generates better constraints than the general-purpose version, we use Figure 9 to show a comparison between two strategies. Here we use the total number of symbolic states (i.e., the input space of all relational operators that introduce symbolic states) to approximate the complexity of constraints. Both the assignment operator $\sigma: \mathbb{A} \times \mathbb{I} \rightarrow \mathbb{B}$ and the readiness operator $\delta: \mathbb{N} \times \mathbb{A} \times \mathbb{T} \rightarrow \mathbb{B}$ introduce symbolic states. Even though the size of the symbolic states generated by the readiness operator can grow as the size of the tree $n$, the number of attributes $q$, and the number of slots $k$ increase, it's still bounded by a polynomial growth. In particular, the domain-specific encoding generates a maximum of $\left(1+n^{2}\right) \cdot q \cdot k$ symbolic states, which is more compact and less complex than the exponential number generated by general-purpose encoding.

\section{EVALUATION}

In this section, we describe the results of the experimental evaluation, which is designed to answer the following key research questions:

(1) (Expressiveness) Is HecAte's tree (visitor/traversal/trace) language expressive enough? In particular, can it express prevailing tree traversal synthesis problems and solve them?

(2) (Performance) What is the performance of synthesized traversals, compared to those generated by state-of-the-art traversal synthesizers?

(3) (Flexibility) Can Hecate be extended to explore traversals of different design choices?

(4) (Efficiency) What is the benefit of the domain-specific encoding compared to general-purpose encoding?

For all experiments, HecATE requires user-provided attribute grammar, a symbolic traversal and an initial example tree as input, and outputs a concrete traversal in tree traversal language $\mathcal{L}_{t}$.

\subsection{Comparison against Grafter}

We first compare HecAte against Grafter [41], the state-of-the-art tree traversal synthesizer based on static dependence analysis. In particular, GRAFTER builds access automata that summarises dependency relations for tree visitors, and synthesizes tree traversals using a deterministic algorithm. We adapt the original benchmark set from GRAFTER, which contains five representative tree traversal synthesis problems from real-world applications. Since GrAFTER benchmarks are written in $\mathrm{C}++$, we also implement a code generator for converting concrete traversals synthesized by HecATE into corresponding $\mathrm{C}++$ versions through syntax-directed translation. To study the benefit of domain-specific encoding discussed in Section 5, we also implement general-purpose encoding discussed in Section 4.2, which we denote as HecATE ${ }^{\mathbb{G}}$.

Efficiency and Expressiveness. Table 2 shows the results of the comparison. In particular, HecATE supports all 5 benchmarks from GRAFTER and successfully synthesizes the correct solutions (i.e., traversals that are semantically equivalent to the ones generated by Grafter.) within 5.9 seconds on average. Specifically, Hecate yields an averaged speed-up of 3.1 $\times$ compared to HeCATE ${ }^{\mathbb{G}}$ and $8.0 \times$ compared to Grafter. The evaluation shows that Hecate's tree language is expressive to support a variety of tree traversal
Table 2: Comparison between Grafter, Hecate and Hecate ${ }^{\mathbb{G}}$ (with general-purpose encoding). The table shows total synthesis time (synthesis + verification) in second.

\begin{tabular}{|c|c|c|c|c|}
\hline Benchmark & \# of Rules & GrAFTER & HECATE & HECATE $^{\mathbb{G}}$ \\
\hline \hline BinaryTree & 16 & 2.6 & 1.1 & 3.2 \\
\hline FMM & 14 & 7.6 & 1.0 & 1.6 \\
\hline Piecewise & 12 & 12.6 & 2.1 & 3.1 \\
\hline AST & 136 & 151.7 & 20.6 & 73.4 \\
\hline RenderTree & 50 & 62.0 & 4.1 & 10.1 \\
\hline
\end{tabular}

applications. Furthermore. the comparison between Hecate and HecAte $^{G}$ also demonstrates the benefits of domain-specific encoding.

Performance. To evaluate the performance of the synthesized traversals, we directly adopt the workload from GrAFTER. Since our symbolic traversals are written in a way to "fuse" tree visitors whenever possible, like GrAFTER, the performance of our synthesized traversals are almost identical to the ones generated by GrAFTER. However, unlike GrAFter that uses a deterministic algorithm for generating one unique solution for each benchmark, the tree language enables HecATE to flexibly explore various traversals of different design choices, some of which lead to dramatic performance speed-up. In what follows, we elaborate on the details using a case study from one of GRAFTER's benchmarks: RenderTree. ${ }^{7}$

Usability. To further minimize user effort, we implement a variant $\operatorname{HecATE}^{\mathbb{A}}$ that incorporates an auto-tuner that can automatically search for useful symbolic traversals during synthesis. In particular, the user only has to provide attribute grammar, and HecATE ${ }^{\mathbb{A}}$ will construct the example trees and initiate an outer loop that searches for a symbolic traversal that ensures correctness of its corresponding synthesized concrete traversal. Our experimental results indicate HecATE ${ }^{\mathbb{A}}$ can solve four GrAfter benchmarks as fast as HecATE; for the AST benchmark with complex symbolic traversals, it takes HecATE ${ }^{\mathbb{A}}$ more than 30 mins to find a solution. We show that it is possible to get rid of more manual inputs for Hecate using a simple auto-tuner.

\subsection{Case Study: RenderTree}

In the RenderTree benchmark, a document tree consists of a list of pages containing nested horizontal and vertical containers with concrete elements as leaf nodes (e.g., text boxes, images, and itemized lists). A total of five rendering passes compute various visual attributes: (1) resolving flexible widths, (2) resolving relative widths, (3) computing heights, (4) propagating font styles, and (5) finalizing positions of elements. Each pass potentially depends on the attributes computed by previous passes.

Unlike GRAFTER, which only generates one unique traversal that fuses tree visitors whenever possible, HEcate offers a number of design choices. For instance, tree nodes frequently visit their children, which can be modeled using either linked lists or vectors. Moreover, when the children have no dependencies between themselves, the user may parallelize the computations using the

\footnotetext{
${ }^{7}$ See Appendix A for a detailed case study for another benchmark: AST.
} 


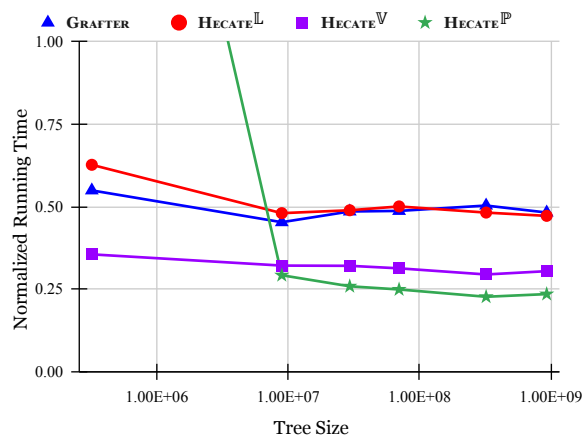

Figure 11: Running time of fused traversals compared to the unfused baseline.

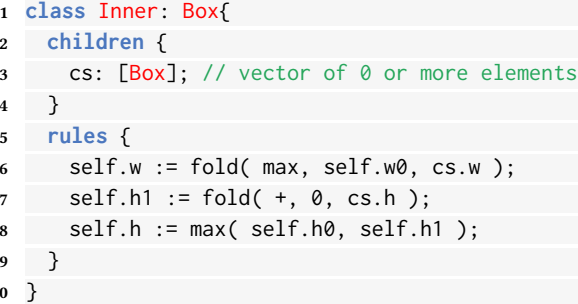

Figure 12: Class definitions in HECATE for rendering tree example, optimized with vector data structure. Only key refactorings are listed.

$\langle$ parallel $\rangle$ construct in the symbolic traversal. Then, HecATE can verify the absence of inter-dependency between children and generate a parallel scheduling over the list of children. Here, we evaluate the performance of the following variants: 1). HecATE $^{\mathbb{L}}$ : sequential linked-list-based traversal 2). HecATE $^{\nabla}$ : sequential vector-based traversal 3). HecAte ${ }^{\mathbb{P}}$ : parallel vector-based traversal. Figure 11 summarizes the comparison with GRAFTER. Here, each line corresponds to one of the variants. The $x$-axis shows the tree size and the workload is directly adopted from the GRAFTER paper. The $y$-axis shows normalized running time over the unfused baseline, averaged over 10 trials.

Linked-List-Based Traversal. Due to limitation of Grafter's static analysis, it only supports linked list for modeling variable-length arrays of children. The HECATE ${ }^{\mathbb{L}}$ variant uses the same linked list data structure, and is able to synthesize a schedule that is semantically equivalent to Grafter's fused traversal. Specifically, HeCATE ${ }^{\mathbb{L}}$ achieves competitive performance against GRAFTER, where both candidates get more than $50 \%$ running time reduction over the unfused traversal.

Sequential Vector-Based Traversal. The traversals in real-world compilers like Clang [37] leverage vectors for iterating children. Because the vector-based layout typically leads to better cache locality and reduces the number of dynamic dispatching due to virtual functions, it is crucial for a traversal synthesizer to explore different design choices. However, GrAFTER does not support vector-based representation due to limitation in its static analysis. On the other

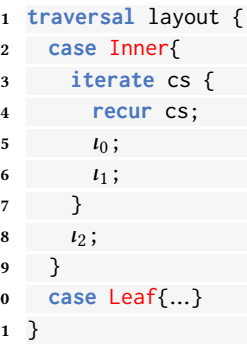

\section{(a) symbolic}

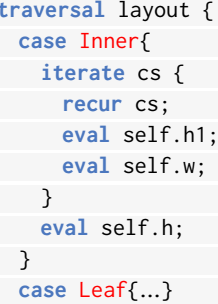

(b) concrete

Figure 13: Symbolic and concrete tree traversals for rendering tree example, optimized with vector data structure. Only key refactorings are listed.

hand, as shown in Figure 12 and Figure 13, it only takes $\operatorname{HecAte}^{\mathbb{V}}$ a few lines of changes to refactor a linked-list-based traversal to its vector-based version. In particular, HecATE ${ }^{\mathbb{V}}$ achieves around $70 \%$ running time reduction and almost $40 \%$ speed-up over GRAFTER's fused traversal.

Parallel Vector-Based Traversal. As Grafter tacitly assumes that fusion opportunities should be exploited whenever possible, it's designed to reduce the number of tree node visits. This heuristic, despite being effective in some scenarios, may prevent further optimizations and lead to sub-optimal traversals in terms of overall running time.

Consider the fused example shown in Figure 14(b): the fused loop iterates over the children to call a traversal function $c->$ fusedCalc $($ ) before updating the running maximum for certain values. Assuming that each c->fusedCalc() is independent from each other, we can "de-fuse" the for loop into two: as shown in Figure 14(c) the first loop is decomposed into parallel traversals, and the second loop updates the running maximum in a sequential fashion. Although the "de-fused" traversal yields a higher number of node visits, it can benefit from parallel execution if the cost of children traversal calls far outweighs the cost of the sequential second visit. This example shows how unconditionally fusing computations might prevent fine-grained optimizations.

As shown in Figure 11, as the tree size grows, the speed-up brought by the parallel variant HecATE ${ }^{\mathbb{P}}$ gradually overcomes its overhead, bringing an additional $23 \%$ improvement over the sequential vector-based variant HecATE ${ }^{\mathbb{V}}$.

The evaluation shows that, with minimal effort, HecATE can effectively explore traversals of different design choices.

\subsection{Synthesizing Layout Engine in FTL}

To show the advantages of our domain-specific encoding, we compare HecATE against FTL [32], a synthesizer specialized for layout engines. In particular, FTL introduces a Prolog-style declarative language for expressing partial schedules with holes. After that, FTL devises a sophisticated synthesis algorithm that leverages Prolog's unification algorithm for effectively generating the schedule as a composition of parallel tree traversals.

Benchmarks. Since FTL is not actively maintained anymore, we can only run it on three variants of attribute grammars (i.e., CSS 
attribute grammar onto a sequence of traversals is known to be NP-hard [19], though polynomial-time scheduling algorithms for restricted classes of grammars exist [32]. However, these restricted classes have not been classified or well-studied.

Constraint solving based on satisfiability modulo theories [33] has become a powerful tool for program analysis as practical, highperformance solvers have become available [2, 18, 21]. Solver-based verification and synthesis tools have a long and rich history in programming languages community [27, 42, 43]. Traditional solveraided tools use a custom constraint solver or manually translate problems into constraints for a specific existing solver. Solver-aided domain-specific languages [45, 47] instead automatically generate solver constraints based on symbolic execution and custom language extensions. For example, RosEtTe [46] uses Racket's metaprogramming features to provide a high-level interface to several solvers. Hecate is build atop Rosette, but uses its trace language to abstract over the low-level features presented in generic RosETTE constraints and significantly improves runtime.

\section{CONCLUSION}

We propose Hecate, a novel framework for synthesizing tree traversals. The core of HecATE is a domain-specific symbolic compilation strategy for tree traversal synthesis that maintains the engineering advantages of solver-aided language, yet achieves better performance. The evaluation shows that HECATE's tree language is expressive as it supports traversals from all GRAFTER benchmarks and complex features in layout engines. HecATE's domain-specific symbolic compilation is efficient as it achieves $3 \times$ speed-up compared to general-purpose symbolic compilation. Finally, Our case analysis shows that HEcATE can explore traversals of different design choices with simple modifications.

\section{ACKNOWLEDGMENTS}

This work has been supported in part by the ACI OAC-1535191, FMitF CCF-1918027, OIA-1936731, SaTC-1908494, the Intel and NSF joint research center for Computer Assisted Programming for Heterogeneous Architectures (CAPA NSF CCF-1723352), the CONIX Research Center, one of six centers in JUMP, a Semiconductor Research Corporation (SRC) program sponsored by DARPA CMU 1042741-394324 AM01, grants from DARPA FA8750-14-C-0011 and DARPA FA8750-16-2-0032, the Google faculty award, as well as gifts from Adobe, Facebook, Google, Intel, and Qualcomm.

\section{A CASE STUDY: AST}

Compilers routinely traverse abstract syntax trees (ASTs) to perform program transformation and validation. The AST benchmark models a simple imperative language with variable assignments, arithmetic expressions, decrement and increment statements, conditional statements, and functions. The benchmark further implements a total of six de-sugaring and optimization passes: 1) desugaring decrement statements, 2) de-sugaring increment statements, 3) constant propagation, 4) replacement of variable references to constants, 5) constant folding, and 6) elimination of unreachable branches.

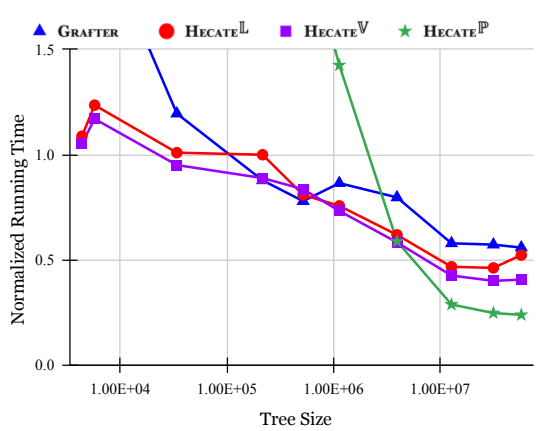

Figure 16: Running time of fused traversals compared to the unfused baseline.

Similar to the RenderTree benchmark, we evaluate the performance of three variants of HecATE: 1). HecATE ${ }^{\mathbb{L}}$ : sequential linkedlist-based traversal 2). HecATE ${ }^{\mathbb{V}}$ : sequential vector-based traversal 3). Hecate ${ }^{\mathbb{P}}$ : parallel vector-based traversal. Figure 16 summarizes the comparison with GRAFTER. Each line corresponds to one of the variants. The $x$-axis shows the tree size, while the $y$-axis shows normalized running time over the unfused baseline, averaged over 10 trials.

Overall, the linked-list based traversal HecATE ${ }^{\mathbb{L}}$ achieves around $50 \%$ running time reduction compared to unfused baseline, which is similar to GRAFTER fused traversal. However, the choice of linked lists for representing lists of statements is not so much a necessity as a limitation from Grafter's static analysis. Hecate $\mathbb{V}$ in contrast, lets us replace the underlying data structure with vectors with minimal code modification, leading to a further $10 \%$ reduction in running time. Furthermore, HecATE $\mathbb{P}^{\mathbb{P}}$ is able to take advantage of the data-independency between optimization passes on different AST functions. Although there is ineivitable overhead when the parallel schedules synthesized by HecATE $\mathbb{P}^{\mathbb{2}}$ are evaluated on smaller trees, the performance gains gradually overcome the overhead, and result in over $75 \%$ running time reduction over the unfused baseline.

\section{REFERENCES}

[1] Greg J. Badros, Alan Borning, Kim Marriott, and Peter J. Stuckey. 1999. Constraint Cascading Style Sheets for the Web. In Proceedings of the 12th Annual ACM Symposium on User Interface Software and Technology (UIST'15). ACM, New York, NY, USA, 73-82. https://doi.org/10.1145/320719.322588

[2] Clark Barrett, Christopher L. Conway, Morgan Deters, Liana Hadarean, Dejan Jovanović, Tim King, Andrew Reynolds, and Cesare Tinelli. 2011. CVC4. In Proceedings of the 23rd International Conference on Computer Aided Verification (Snowbird, UT) (CAV'11). Springer-Verlag, Berlin, Heidelberg, 171-177. http: //dl.acm.org/citation.cfm?id=2032305.2032319

[3] Jeffrey P. Bigham. 2014. Making the Web Easier to See with Opportunistic Accessibility Improvement. In Proceedings of the 27th Annual ACM Symposium on User Interface Software and Technology (Honolulu, Hawaii, USA) (UIST '14). ACM, New York, NY, USA, 117-122. https://doi.org/10.1145/2642918.2647357

[4] Alan Borning, Richard Lin, and Kim Marriott. 1997. Constraints for the Web. In Proceedings of the Fifth ACM International Conference on Multimedia (Seattle, Washington, USA) (MULTIMEDIA '97). ACM, New York, NY, USA, 173-182. https: //doi.org/10.1145/266180.266361

[5] Bert Bos. 2016. CSS 2.2: Collapsing Margins. https://tinyurl.com/j66mfru.

[6] Bert Bos. 2016. CSS 2.2: Floats. https://tinyurl.com/ssn8rco.

[7] Bert Bos, Tantek Çelik, Ian Hickson, and Håkon Wium Lie. 2011. CSS 2.1: Collapsing Margins. https://tinyurl.com/rspsl2j.

[8] Bert Bos, Tantek Çelik, Ian Hickson, and Håkon Wium Lie. 2011. CSS 2.1: Floats. https://tinyurl.com/s67ebaa. 
[9] Bert Bos, Håkon Wium Lie, Chris Lilley, and Ian Jacobs. 1998. CSS 2: Collapsing Margins. https://tinyurl.com/seb5h92.

[10] Bert Bos, Håkon Wium Lie, Chris Lilley, and Ian Jacobs. 1998. CSS 2: Floats. https://tinyurl.com/vbt29em.

[11] Browserling. 2018. https://www.browserling.com/

[12] Browsershots. 2018. http://browsershots.org/

[13] Browserstack. 2018. https://www.browserstack.com/screenshots

[14] Matt Brubeck. 2014. Incorrect layout of element following a float, involving margins. https://github.com/servo/servo/issues/4307.

[15] Kartik Chandra and Rastislav Bodík. 2018. Bonsai: synthesis-based reasoning for type systems. Proc. ACM Program. Lang. 2, POPL (2018), 62:1-62:34.

[16] S. R. Choudhary, M. R. Prasad, and A. Orso. 2012. CrossCheck: Combining Crawling and Differencing to Better Detect Cross-browser Incompatibilities in Web Applications. In 2012 IEEE Fifth International Conference on Software Testing, Verification and Validation. 171-180. https://doi.org/10.1109/ICST.2012.97

[17] Ravi Chugh, Brian Hempel, Mitchell Spradlin, and Jacob Albers. 2016. Programmatic and Direct Manipulation, Together at Last. In Proceedings of the 37th ACM SIGPLAN Conference on Programming Language Design and Implementation (Santa Barbara, CA, USA) (PLDI '16). ACM, New York, NY, USA, 341-354. https://doi.org/10.1145/2908080.2908103

[18] Leonardo De Moura and Nikolaj Bjørner. 2008. Z3: An Efficient SMT Solver. In Proceedings of the Theory and Practice of Software, 14th International Conference on Tools and Algorithms for the Construction and Analysis of Systems (Budapest, Hungary) (TACAS'08/ETAPS'08). Springer-Verlag, Berlin, Heidelberg, 337-340. http://dl.acm.org/citation.cfm?id=1792734.1792766

[19] Joost Engelfriet and Gilberto Filè. 1982. Simple multi-visit attribute grammars Journal of computer and system sciences 24, 3 (1982), 283-314. http://doc.utwente. $\mathrm{nl} / 69001 /$

[20] Yu Feng, Ruben Martins, Osbert Bastani, and Isil Dillig. 2018. Program synthesis using conflict-driven learning. In Proceedings of the 39th ACM SIGPLAN Conference on Programming Language Design and Implementation, PLDI 2018, Philadelphia, PA, USA, Fune 18-22, 2018, Jeffrey S. Foster and Dan Grossman (Eds.). ACM, 420-435.

[21] LLC Gurobi Optimization. 2019. Gurobi Optimizer Reference Manual. http: //www.gurobi.com

[22] Sylvain Halle, Nicolas Bergeron, Francis Guerin, and Gabriel Le Breton. 2015 Testing Web Applications Through Layout Constraints. In 2015 IEEE 8th International Conference on Software Testing, Verification and Validation (ICST). 1-8. https://doi.org/10.1109/ICST.2015.7102635

[23] Osamu Hashimoto and Brad A. Myers. 1992. Graphical Styles for Building Interfaces by Demonstration. In Proceedings of the 5th Annual ACM Symposium on User Interface Software and Technology (Monteray, California, USA) (UIST '92). ACM, New York, NY, USA, 117-124. https://doi.org/10.1145/142621.142635

[24] IBM. 2016. The CPLEX Optimizer. https://www.ibm.com/analytics/cplexoptimizer/.

[25] Anantha Keesara. 2007. Bug 15662 - layout is coming down because of style 'float:left' of < div>. https://bugs.webkit.org/show bug.cgi?id=15662.

[26] Donald E. Knuth. 1968. Semantics of Context-Free Languages. In In Mathematical Systems Theory. 127-145.

[27] K. Rustan M. Leino. 2010. Dafny: An Automatic Program Verifier for Functional Correctness. In Proceedings of the 16th International Conference on Logic for Programming, Artificial Intelligence, and Reasoning (Dakar, Senegal) (LPAR'10) Springer-Verlag, Berlin, Heidelberg, 348-370. http://dl.acm.org/citation.cfm?id= 1939141.1939161

[28] Sonal Mahajan, Negarsadat Abolhassani, Phil McMinn, and William G.J. Halfond. 2018. Automated Repair of Mobile Friendly Problems in Web Pages. In International Conference on Software Engineering (ICSE 2018). ACM, 140-150.

[29] Sonal Mahajan, Abdulmajeed Alameer, Phil McMinn, and William G. J. Halfond. 2017. Automated Repair of Layout Cross Browser Issues Using Search-based Techniques. In Proceedings of the 26th ACM SIGSOFT International Symposium on Software Testing and Analysis (Santa Barbara, CA, USA) (ISSTA 2017). ACM, New York, NY, USA, 249-260. https://doi.org/10.1145/3092703.3092726

[30] S. Mahajan, A. Alameer, P. McMinn, and W. G. J. Halfond. 2018. Automated Repair of Internationalization Presentation Failures in Web Pages Using Style Similarity Clustering and Search-Based Techniques. In 2018 IEEE 11th International Conference on Software Testing, Verification and Validation (ICST). 215-226. https://doi.org/10.1109/ICST.2018.00030

[31] A. Mesbah and M. R. Prasad. 2011. Automated cross-browser compatibility testing. In 2011 33rd International Conference on Software Engineering (ICSE). 561-570. https://doi.org/10.1145/1985793.1985870

[32] Leo A. Meyerovich, Matthew E. Torok, Eric Atkinson, and Rastislav Bodik. 2013. Parallel Schedule Synthesis for Attribute Grammars. In Proceedings of the 18th ACM SIGPLAN Symposium on Principles and Practice of Parallel Programming (Shenzhen, China) (PPoPP '13). ACM, New York, NY, USA, 187-196. https: //doi.org/10.1145/2442516.2442535

[33] Greg Nelson and Derek C. Oppen. 1980. Fast Decision Procedures Based on Congruence Closure. F. ACM 27, 2 (April 1980), 356-364. https://doi.org/10. $1145 / 322186.322198$
[34] Pavel Panchekha, Adam T. Geller, Michael D Ernst, Zachary Tatlock, and Shoaib Kamil. 2018. Verifying That Web Pages Have Accessible Layout (PLDI'18). https: //doi.org/10.1145/3192366.3192407

[35] Pavel Panchekha and Emina Torlak. 2016. Automated Reasoning for Web Page Layout. In Proceedings of the 2016 ACM SIGPLAN International Conference on Object-Oriented Programming, Systems, Languages, and Applications (Amsterdam, Netherlands) (OOPSLA 2016). ACM, New York, NY, USA, 181-194. https://doi. org $/ 10.1145 / 2983990.2984010$

[36] Phitchaya Mangpo Phothilimthana, Tikhon Jelvis, Rohin Shah, Nishant Totla, Sarah Chasins, and Rastislav Bodik. 2014. Chlorophyll: Synthesis-aided Compiler for Low-power Spatial Architectures. In Proceedings of the 35th ACM SIGPLAN Conference on Programming Language Design and Implementation (Edinburgh, United Kingdom) (PLDI '14). ACM, New York, NY, USA, 396-407. https://doi. org/10.1145/2594291.2594339

[37] The Clang Project. 2021. Clang: a C language family frontend for LLVM. https: //clang.llvm.org.

[38] The Servo Parallel Browser Engine Project. 2018. Rendering issue of styled buttons. https://github.com/servo/servo/issues/18991.

[39] Shauvik Roy Choudhary, Husayn Versee, and Alessandro Orso. 2010. WEBDIFF: Automated Identification of Cross-browser Issues in Web Applications. In Proceedings of the 2010 IEEE International Conference on Software Maintenance (ICSM '10). IEEE Computer Society, Washington, DC, USA, 1-10. https: //doi.org/10.1109/ICSM.2010.5609723

[40] Laith Sakka, Kirshanthan Sundararajah, and Milind Kulkarni. 2017. TreeFuser: a framework for analyzing and fusing general recursive tree traversals. Proc. ACM Program. Lang. 1, OOPSLA (2017), 76:1-76:30.

[41] Laith Sakka, Kirshanthan Sundararajah, Ryan R. Newton, and Milind Kulkarni. 2019. Sound, fine-grained traversal fusion for heterogeneous trees. In Proceedings of the 40th ACM SIGPLAN Conference on Programming Language Design and Implementation, PLDI 2019, Phoenix, AZ, USA, fune 22-26, 2019, Kathryn S. McKinley and Kathleen Fisher (Eds.). ACM, 830-844.

[42] Eric Schkufza, Rahul Sharma, and Alex Aiken. 2013. Stochastic Superoptimization. In Proceedings of the Eighteenth International Conference on Architectural Support for Programming Languages and Operating Systems (Houston, Texas, USA) (ASPLOS '13). ACM, New York, NY, USA, 305-316. https: //doi.org/10.1145/2451116.2451150

[43] Armando Solar-Lezama. 2008. Program Synthesis by Sketching. Ph.D. Dissertation. Berkeley, CA, USA. Advisor(s) Bodik, Rastislav. AAI3353225.

[44] Ivan E. Sutherland. 1964. Sketch Pad a Man-machine Graphical Communication System. In Proceedings of the SHARE Design Automation Workshop (DAC '64). ACM, New York, NY, USA, 6.329-6.346. https://doi.org/10.1145/800265.810742

[45] Emina Torlak and Rastislav Bodik. 2013. Growing Solver-aided Languages with Rosette. In Proceedings of the 2013 ACM International Symposium on New Ideas, New Paradigms, and Reflections on Programming \& Software (Indianapolis, Indiana, USA) (Onward! 2013). ACM, New York, NY, USA, 135-152. https://doi.org/10. 1145/2509578.2509586

[46] Emina Torlak and Rastislav Bodik. 2014. A Lightweight Symbolic Virtual Machine for Solver-aided Host Languages. In Proceedings of the 35th ACM SIGPLAN Conference on Programming Language Design and Implementation (Edinburgh, United Kingdom) (PLDI '14). ACM, New York, NY, USA, 530-541. https://doi.org/10.1145/2594291.2594340

[47] Richard Uhler and Nirav Dave. 2014. Smten with Satisfiability-based Search. In Proceedings of the 2014 ACM International Conference on Object Oriented Programming Systems Languages \& Applications (Portland, Oregon, USA) (OOPSLA '14). ACM, New York, NY, USA, 157-176. https://doi.org/10.1145/2660193.2660208

[48] Christopher J. van Wyk. 1982. A High-Level Language for Specifying Pictures. ACM Trans. Graph. 1, 2 (April 1982), 163-182. https://doi.org/10.1145/357299. 357303

[49] Thomas A. Walsh, Gregory M. Kapfhammer, and Phil McMinn. 2017. Automated Layout Failure Detection for Responsive Web Pages Without an Explicit Oracle. In Proceedings of the 26th ACM SIGSOFT International Symposium on Software Testing and Analysis (Santa Barbara, CA, USA) (ISSTA 2017). ACM, New York, NY, USA, 192-202. https://doi.org/10.1145/3092703.3092712

[50] T. A. Walsh, P. McMinn, and G. M. Kapfhammer. 2015. Automatic Detection of Potential Layout Faults Following Changes to Responsive Web Pages (N). In 2015 30th IEEE/ACM International Conference on Automated Software Engineering (ASE). 709-714. https://doi.org/10.1109/ASE.2015.31

[51] Leland Wilkinson. 2005. The Grammar of Graphics (Statistics and Computing). Springer-Verlag New York, Inc., Secaucus, NJ, USA.

[52] Brad Vander Zanden and Brad A. Myers. 1991. The Lapidary Graphical Interface Design Tool. In Proceedings of the SIGCHI Conference on Human Factors in Computing Systems (New Orleans, Louisiana, USA) (CHI '91). ACM, New York, NY, USA, 465-466. https://doi.org/10.1145/108844.109005 